\title{
Highly selective direct aldol reaction organocatalyzed by $(S)$-BINAM-L-prolinamide and benzoic acid using $\alpha$-chalcogen-substituted ketones as donors
}

\author{
Gabriela Guillena, María del Carmen Hita, and Carmen Nájera* \\ Departamento de Química Orgánica and Instituto de Síntesis Orgánica (ISO), \\ Universidad de Alicante, Apartado 99, 03080 Alicante, Spain \\ E-mail: cnajera@ua.es
}

Dedicated to Professor J. Bosch on occasion of his 60th birthday

\begin{abstract}
Recoverable $(S)$-BINAM-L-prolinamide in combination with benzoic acid catalyzes and accelerates the direct aldol reaction between several $\alpha$-chalcogen-substituted ketones and $p$ nitrobenzaldehyde in different solvents, including water. Choosing conveniently the aldol donor, solvent and conditions, it is possible to obtain mainly one of the two possible regioisomers with good diastero- and enantioselectivity. Thus, $\alpha$-hydroxy and $\alpha$-alkoxyacetones give mainly syn/anti regioisomers, whereas $\alpha$-(methylsulphanyl)acetone affords the iso-aldol in $93 \%$ ee. In the case of $\alpha$-hydroxy and $\alpha$-methoxyacetone the anti diasteromer is obtained with up to $84 \%$ ee. However, $\alpha$-benzyloxyacetone yields mainly the syn-diasteroisomer in $85 \%$ ee.
\end{abstract}

Keywords: Asymmetric synthesis, aldol reaction, diols, organocatalysis, enantioselectivity

\section{Introduction}

Asymmetric organocatalysis ${ }^{1}$ has become one of the most powerful tools in synthetic chemistry to perform the enantioselective synthesis of organic compounds through $\mathrm{C}-\mathrm{C}$ bond formation reactions, as well as in C-heteroatom bond formation processes. ${ }^{2}$ The application of this "enzyme mimics"3 has been specially useful to perform the asymmetric aldol reaction. ${ }^{4}$ In nature, this reaction is perfectly catalyzed by enzymes (class I or class II aldolases) with excellent stereocontrol, water being the reaction media. Proline ${ }^{5}$ and its derivatives ${ }^{6}$ have shown to be excellent catalysts for the asymmetric aldol reaction in organic solvents, the success of organocatalysts for this transformation in water being only very recently achieved. ${ }^{7}$ One of the challenges for the direct aldol reaction is the simultaneous control of the regio-, diastero-, and enantioselectivity when unsymmetrical ketones are used (Scheme 1). 


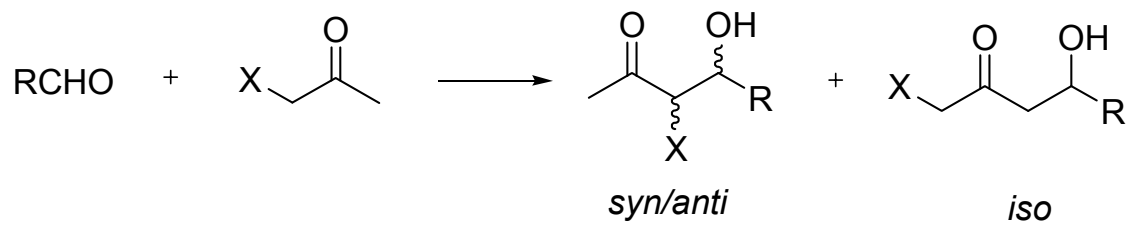

Scheme 1. Aldol reaction between unsymmetrical ketones and aldehydes.

Especially attractive is the use of functionalized ketones, which can lead to the synthesis of polyfunctionalized molecules with high degree of selectivity. However, only the coupling of $\alpha$ hydroxyketone ${ }^{8,5 a, 60}$ as aldol nucleophilic donor with aldehydes has been extensively studied, the use of other $\alpha$-functionalized ketones remaining testimonial. ${ }^{9}$ Recently, it has been reported that the use of BINAM-prolinamides such as $\mathbf{1}$ and their use for the direct aldol reaction between aldehydes and alkyl ${ }^{10,11}$ and alkoxy ketones ${ }^{12}$ provide the expected products with high regio-, diastereo- and enantioselectivity. In addition, this type of BINAM-prolinamides can be recovered after simple acid-base extractive work-up. Moreover, we have found that a high acceleration of the reaction was achieved with alkyl ketones when the reaction was co-catalyzed by an acid such as benzoic acid, without becoming detrimental to the previously obtained results. This effect was not observed when L-Pro was used as organocatalyst. ${ }^{13}$ Here, we describe the application of these new found reaction conditions to the aldol reaction between several $\alpha$-chalcogen-functionalized ketones and 4-nitrobenzaldehyde, as well as their applicability in organic and in aqueous media.

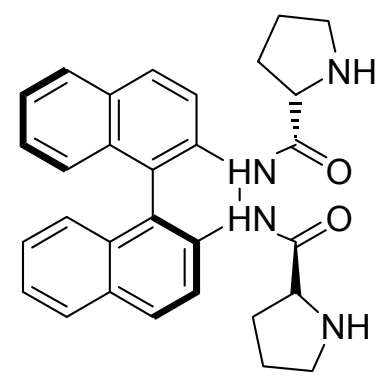

1, $\left(S_{a}\right)$-BINAM-L-Pro

\section{Results and Discussion}

Catalyst $\left(S_{a}\right)$-BINAM-L-Pro (1) was synthesized as previously described ${ }^{10}$ starting form 2,2'diamino-1,1'-binaphtalene (BINAM) and L-proline. ${ }^{14}$ Initial studies of the acid co-catalyzed aldol reaction between $\alpha$-chalcogen-functionalized ketones (Scheme 2 and Table 1), were performed using hydroxyacetone ${ }^{15}$ as aldol donor and representative 4-nitrobenzaldehyde as electrophilic counterpart. As it has been previously reported, ${ }^{12}$ the best solvent to carry out the reaction with $\alpha$-hydroxyacetone is DMSO, using $20 \mathrm{~mol} \%$ of catalyst 1 . When the reaction was 
performed in DMSO at $0{ }^{\circ} \mathrm{C}$ in the absence of acid, the expected product was obtained after $1 \mathrm{~d}$ as a mixture of the three possible regioisomers $\mathbf{2 a / 3 a}(4: 1)$, the major isomer anti-2a being achieved with a $80 \%$ ee (Table 1 , entry 1$).{ }^{12}$ When the reaction was done in the presence of 40 mol\% of benzoic acid under the same reaction conditions, the reaction was much faster $(4 \mathrm{~h})$ and the products were obtained with higher regioselectivity (50:1) and with similar diastereoselectivity but lower ee (Table 1, entry 2). When the solvent was changed to DMF similar regio- and diastereoselectivity was obtained, but in shorter reaction time when benzoic acid was present. However, slightly lower ee was obtained in DMF than in DMSO. The addition of water to DMF had a strong negative effect in the achieved ee (Table 1, compare entries 3 to 6). Thus, DMF was chosen as solvent to perform the reaction at $-20^{\circ} \mathrm{C}$ in the presence of benzoic acid expecting an increase of the achieved diastero- and enantioselectivity. Unfortunately, although the regio- and diastereoselectivity was maintained, the enantioselectivities decreased (Table 1, compare entries 4 and 7).
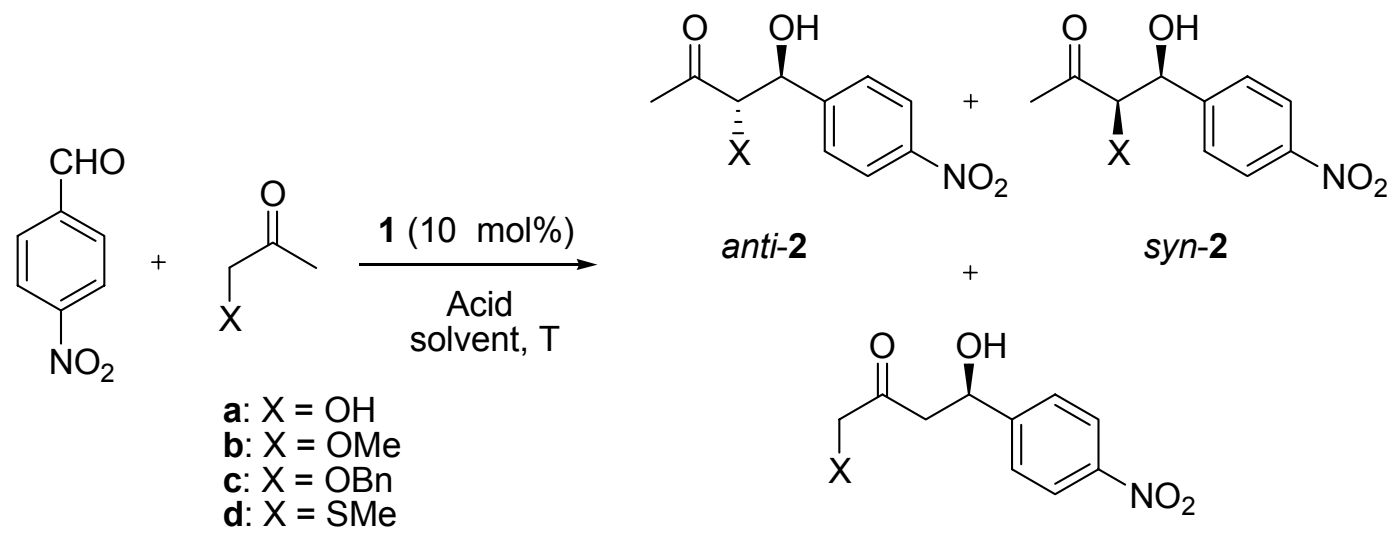

anti-2

syn-2

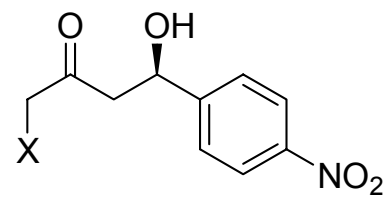

iso-3

Scheme 2. Aldol reaction between $\alpha$-chalcogen functionalized ketones and 4-nitrobenzaldehyde.

As $\mathrm{Cl}_{2} \mathrm{CHCO}_{2} \mathrm{H}$ gave the best enantioselectivity when it was used as co-catalyst in the aldol reaction between acetone and 4-nitrobenzaldehyde, ${ }^{13}$ we decided to use $20 \mathrm{~mol} \%$ of this acid in $\mathrm{DMF}$ at $0{ }^{\circ} \mathrm{C}$. In this case the reaction was over in $2 \mathrm{~h}$ with an excellent regioselectivity (50:1), good diastereoselectivity (3:1) and a $68 \%$ ee for the anti-2a product, comparable to that obtained in DMSO in the presence of benzoic acid (compare entries 2 and 8 in Table 1). It can be concluded that for $\alpha$-hydroxyacetone the presence of a carboxylic acid improves the regioselectivity, up to 50:1, as well as the reaction rate. 
Table 1. Aldol reaction between different $\alpha$-chalcogen substituted ketones and 4nitrobenzaldehyde catalyzed by $\mathbf{1}^{\mathrm{a}}$

\begin{tabular}{|c|c|c|c|c|c|c|c|c|c|c|c|}
\hline \multirow{2}{*}{ Ent. } & \multirow{2}{*}{$\mathrm{X}$} & \multirow{2}{*}{ Solvent } & \multirow{2}{*}{ Acid (\%) } & \multirow{2}{*}{$\begin{array}{c}\mathrm{T} \\
\left({ }^{\circ} \mathrm{C}\right)\end{array}$} & \multirow{2}{*}{$\begin{array}{c}\mathrm{t} \\
(\mathrm{h})\end{array}$} & \multirow{2}{*}{$\begin{array}{l}\text { Yield } \\
(\%)^{b}\end{array}$} & \multicolumn{2}{|c|}{ Isomer ratio $^{c}$} & \multicolumn{3}{|c|}{$e e(\%)^{d}$} \\
\hline & & & & & & & $\begin{array}{l}\text { Regio. } \\
(\mathbf{2} / \mathbf{3})\end{array}$ & $\begin{array}{c}\mathrm{dr} \\
(\text { anti/syn) }\end{array}$ & $\begin{array}{c}\text { anti- } \\
\mathbf{2} \\
\end{array}$ & $\begin{array}{c}\text { syn- } \\
\mathbf{2} \\
\end{array}$ & $\begin{array}{c}i s o- \\
\mathbf{3}\end{array}$ \\
\hline $1^{\mathrm{e}}$ & $\mathrm{OH}^{\mathrm{f}}$ & $\mathrm{DMSO}^{\mathrm{g}}$ & - & 0 & 24 & 96 & $4: 1$ & $5: 2$ & 80 & 23 & 84 \\
\hline 2 & $\mathrm{OH}^{\mathrm{f}}$ & $\mathrm{DMSO}^{\mathrm{g}}$ & $\mathrm{PhCO}_{2} \mathrm{H}(40)$ & 0 & 4 & 97 & $>50: 1$ & $2: 1$ & 65 & 14 & - \\
\hline $3^{\mathrm{e}}$ & $\mathrm{OH}^{\mathrm{f}}$ & $\mathrm{DMF}$ & - & 0 & 96 & 98 & $>50: 1$ & $1: 1$ & 74 & 31 & - \\
\hline 4 & $\mathrm{OH}^{\mathrm{f}}$ & DMF & $\mathrm{PhCO}_{2} \mathrm{H}(40)$ & 0 & 3 & 99 & $>50: 1$ & $3: 2$ & 58 & 26 & - \\
\hline 5 & $\mathrm{OH}^{\mathrm{f}}$ & $\mathrm{DMF} / \mathrm{H}_{2} \mathrm{O}^{\mathrm{h}}$ & - & 25 & 24 & 98 & $9: 1$ & $1: 1$ & 19 & 74 & 85 \\
\hline 6 & $\mathrm{OH}^{\mathrm{f}}$ & $\mathrm{DMF} / \mathrm{H}_{2} \mathrm{O}^{\mathrm{h}}$ & $\mathrm{PhCO}_{2} \mathrm{H}(40)$ & 0 & 28 & 85 & $>50: 1$ & $3: 1$ & 13 & 9 & - \\
\hline 7 & $\mathrm{OH}^{\mathrm{f}}$ & DMF & $\mathrm{PhCO}_{2} \mathrm{H}(40)$ & -20 & 8 & 97 & $>50: 1$ & $2: 1$ & 47 & 27 & - \\
\hline 8 & $\mathrm{OH}$ & $\mathrm{DMF}$ & $\begin{array}{c}\mathrm{Cl}_{2} \mathrm{CHCO}_{2} \mathrm{H} \\
\text { (20) }\end{array}$ & 0 & 2 & 98 & $>50: 1$ & $3: 1$ & 68 & 3 & - \\
\hline $9^{\mathrm{e}}$ & $\mathrm{OMe}$ & DMF & - & 0 & 96 & 88 & $5: 1$ & $7: 1$ & 92 & 58 & 80 \\
\hline 10 & $\mathrm{OMe}$ & DMF & $\mathrm{PhCO}_{2} \mathrm{H}(20)$ & 0 & 22 & 99 & $7: 1$ & $5: 1$ & 84 & 36 & 60 \\
\hline $11^{\mathrm{e}}$ & $\mathrm{OMe}$ & $\mathrm{DMF} / \mathrm{H}_{2} \mathrm{O}^{\mathrm{h}}$ & - & 0 & 48 & 99 & $7: 3$ & $6: 1$ & 84 & 55 & 83 \\
\hline 12 & $\mathrm{OMe}$ & $\mathrm{DMF} / \mathrm{H}_{2} \mathrm{O}^{\mathrm{h}}$ & $\mathrm{PhCO}_{2} \mathrm{H}(20)$ & 0 & 25 & 90 & $5: 1$ & $6: 1$ & 81 & 41 & 79 \\
\hline 13 & $\mathrm{OMe}$ & $\mathrm{DMF} / \mathrm{H}_{2} \mathrm{O}^{\mathrm{h}}$ & $\mathrm{PhCO}_{2} \mathrm{H}(20)$ & -20 & 108 & 56 & $5: 1$ & $5: 1$ & 78 & 51 & 83 \\
\hline 14 & $\mathrm{OMe}$ & $\mathrm{H}_{2} \mathrm{O}$ & $\mathrm{PhCO}_{2} \mathrm{H}(20)$ & 0 & 144 & 74 & $12: 1$ & $5: 1$ & 78 & 46 & 70 \\
\hline $15^{\mathrm{e}}$ & OBn & DMF & - & 0 & 120 & 99 & $1: 1.5$ & $1: 2$ & 19 & 88 & 60 \\
\hline 16 & OBn & DMF & $\mathrm{PhCO}_{2} \mathrm{H}(20)$ & 0 & 39 & 98 & $5: 1$ & $1: 12$ & 35 & 85 & 63 \\
\hline 17 & $\mathrm{SMe}^{\mathrm{i}}$ & $\mathrm{DMF} / \mathrm{H}_{2} \mathrm{O}^{\mathrm{h}}$ & - & 0 & 240 & 93 & $>50: 1$ & $1: 1$ & 6 & 2 & - \\
\hline 18 & $\mathrm{SMe}$ & $\mathrm{DMF} / \mathrm{H}_{2} \mathrm{O}^{\mathrm{h}}$ & - & 0 & 120 & 88 & $1: 6$ & $1: 1$ & - & - & 75 \\
\hline 19 & $\mathrm{SMe}$ & $\mathrm{DMF} / \mathrm{H}_{2} \mathrm{O}^{\mathrm{h}}$ & $\mathrm{PhCO}_{2} \mathrm{H}(20)$ & 0 & 30 & 85 & $1: 8$ & $1: 1$ & - & - & 93 \\
\hline 20 & $\mathrm{SMe}$ & $\mathrm{H}_{2} \mathrm{O}$ & $\mathrm{PhCO}_{2} \mathrm{H}(20)$ & 0 & 21 & 89 & $1: 8$ & $1: 1$ & - & - & 93 \\
\hline
\end{tabular}

a The reactions were carried out using 27.6 equiv of ketone per equiv of aldehyde $(0.25 \mathrm{mmol})$ in the presence of $1(10 \mathrm{~mol} \%)$ and benzoic acid (20 mol \%) in $0.5 \mathrm{~mL}$ of solvent, unless otherwise stated.

${ }^{\mathrm{b}}$ Isolated product after column chromatography.

${ }^{\mathrm{c}}$ Determined by ${ }^{1} \mathrm{H}$ NMR of the crude mixture.

${ }^{\mathrm{d}}$ Determined by HPLC.

${ }^{\mathrm{e}}$ See reference 12.

f $20 \mathrm{~mol} \%$ of catalyst 1 was used.

g $1 \mathrm{~mL}$ of DMSO was used.

h $1: 1$ volume mixture.

${ }^{\mathrm{i}}$ L-Proline (20 mol \%) was used as catalysts. 
$\alpha$-Methoxyacetone is a common nucleophile in antibody catalyzed aldol reactions, ${ }^{16}$ therefore it was considered as aldol donor for this organocatalyzed reaction. ${ }^{12}$ When the condensation of this nucleophile and 4-nitrobenzaldehyde was carried out in either neat or aqueous DMF at $0{ }^{\circ} \mathrm{C}$, similar results were obtained although the presence of benzoic acid increased the reaction rates (Table 1, compare entries 9 with 10 and 11 with 12). Then, the temperature was lowered to $-20{ }^{\circ} \mathrm{C}$ in order to increase the obtained enantioselectivies, but unfortunately under these conditions, the reaction was uncompleted (56\% yield) after $108 \mathrm{~h}$, and the products were obtained with slightly lower diastero-, and enantioselectivities (Table 1, entry 13). The acceptable results obtained in aqueous DMF medium, prompted us to carry out the reaction in neat water. In this case the reaction was much slower $(6 \mathrm{~d})$, but the regioselectivity was increased to $12: 1$, the diastero- and enantioselectivies being similar to that obtained in aqueous DMF (Table 1, compare entries 12 and 14).

In our previous studies we found that using $\alpha$-benzyloxyacetone in DMF at $0{ }^{\circ} \mathrm{C}$ after $5 \mathrm{~d}$, the major isomer was the regioisomer iso-3c. ${ }^{12}$ (Table 1, entry 15). Therefore, we studied the acceleration of this reaction when benzoic acid was used as co-catalyst. To our surprise, the regioselectivity was reversed under this conditions (2c/3c ratio, 5:1), the major isomer syn-2c being obtained in $39 \mathrm{~h}$ with a $92 \%$ de and $85 \%$ ee (Table 1, entry 16 ).

Finally, the use of $\alpha$-(methylsulfanyl)acetone as nucleophile in the direct aldol reaction is restricted to the reaction catalyzed by antibodies, ${ }^{16}$ the $i$ so isomer being obtained as major product with good enantioselectivity. To the best of our knowledge this ketone has never been used as nucleophile in an organocatalyzed reaction, therefore we tested proline as catalyst as first approach to carry out this reaction. In aqueous DMF, after $10 \mathrm{~d}$ reaction time, the product mixture was almost composed by the anti-2d and syn-2d in a nearly racemic mixture (Table 1 , entry 17). However, when (S)-BINAM-L-Pro was used as catalyst in aqueous DMF $(1: 1)$ at $0{ }^{\circ} \mathrm{C}$, after $5 \mathrm{~d}$ the main product obtained with a $75 \%$ ee was the iso-3d (Table 1 entry 18). In the presence of $20 \mathrm{~mol} \%$ of benzoic acid, the reaction time was shortened to $30 \mathrm{~h}$, the regioselectivity increased up to 1:8 favoring the iso-3d product, and the enantioselectivity reaching a $93 \%$ ee (Table 1, entry 19). The use of water as the only solvent for this transformation, led mainly to the iso-3d product in only $21 \mathrm{~h}$ with the same regio-, diastereo-, and enantioselectivity as in aqueous DMF (Table 1, compare entries 19 and 20). These results showed the superiority of catalyst $\mathbf{1}$ in this reaction. It seems reasonable to justify the formation of the iso-isomer based on steric reasons.

As conclusion, a great acceleration of the reaction was observed in the aldol reaction catalyzed by $(S)$-BINAM-L-prolinamide $\mathbf{1}$ in the presence of carboxylic acids whereas this influence was not observed for L-proline catalyzed reactions. Furthermore, depending on the $\alpha$ functionalized ketone used as nucleophile in the direct aldol reaction, we were able to obtain the anti-, syn- or iso-isomer as the main product with good enantioselectivity. With respect to the regioselectivity, the bulkier $\alpha$-(methylsulphanyl)acetone favoured the formation of the iso-3d product with $93 \%$ ee, $(S)$-BINAM-L-Pro being superior to L-proline. In the case of $\alpha$-alkoxy substituted ketones, $\alpha$-methoxyacetone gave the highest diasteroselectivity (up to $86 \%$ ), whereas 
$\alpha$-benzyloxyacetone afforded mainly the $s y n-2 \mathrm{c}$ product (up to $92 \%$ de). However, the presence of benzoic acid did not improve the achieved enantioselectivities for the major products.

\section{Experimental Section}

General Procedures. All reactions were carried out under argon. DMF, DMSO and all others reagents were commercially available and used without further purification. $\alpha$-Hydroxyacetone was distilled prior to use. Only the structural most important peaks of the IR spectra (recorded on a Nicolet 510 P-FT) are listed. ${ }^{1} \mathrm{H}$ NMR (300 MHz, $\left.400 \mathrm{MHz}\right)$ and ${ }^{13} \mathrm{C}$ NMR (75 MHz) spectra were obtained on a Bruker AC-300 using $\mathrm{CDCl}_{3}$ as solvent and TMS as internal standard, unless otherwise stated. Optical rotations were measured on a Perkin Elmer 341 polarimeter. HPLC analyses were performed on a Shimadzu LC-10AD and on a JASCO AS 2059 PLUS equipped with a chiral column (detailed for each compound in the main text), using mixtures of nhexane/isopropyl alcohol (IPA) as mobile phase, at $25^{\circ} \mathrm{C}$. High-resolution mass spectra were obtained on a Finnigan VG Platform. HRMS (DIP) were recorded on a Finnigan MAT 95S. Analytical TLC was performed on Schleicher \& Schuell F1400/LS silica gel plates and the spots were visualised under UV light $(\lambda=254 \mathrm{~nm})$. For flash chromatography we employed Merck silica gel $60(0.040-0.063 \mathrm{~mm})$.

\section{General procedure for the aldol reaction.}

To a solution of 4-nitrobenzaldehyde $(37.8 \mathrm{mg}, 0.25 \mathrm{mmol})$, in dry solvent $(0.5 \mathrm{~mL})$ under argon was added the corresponding ketone $(6.9 \mathrm{mmol})$. To the resulting solution the catalyst was added (10 mol\%, with exception of $\alpha$-hydroxyacetone in this case a $20 \mathrm{~mol} \%$ of catalysts 1 was added) in one portion, and the resulting mixture was stirred at the corresponding temperature. The reaction was monitored by ${ }^{1} \mathrm{H}$ NMR spectroscopy and quenched with $6 \mathrm{M} \mathrm{HCl}(5 \mathrm{~mL})$ and ethyl acetate $(5 \mathrm{~mL})$. The mixture was stirred vigorously for $10 \mathrm{~min}$. The emulsion was separated $(3 \times 5$ $\mathrm{mL}$ sat. $\mathrm{NaCl}$ ) and the aqueous phase treated with a sat. $\mathrm{NaOH}$ solution until $\mathrm{pH}>9$ and then ethyl acetate was added $(3 \times 15 \mathrm{~mL})$. The organic layer was separated, dried $\left(\mathrm{MgSO}_{4}\right)$ and evaporated recovering pure catalyst (ca. 85\%). The organic phase from the acidic work up was dried $\left(\mathrm{MgSO}_{4}\right)$ and evaporated to dryness. The residue was purified by flash chromatography yielding pure aldol products. Spectroscopic and physical data for the aldol products follow:

3,4-Dihydroxy-4-(4'-nitrophenyl)- 2-butanone (anti-/syn-2a). ${ }^{16 \mathrm{~b}}$ HPLC (Chiralpak AD; 1.3 $\mathrm{mL} / \mathrm{min} ; 96: 4 \mathrm{Hex} / \mathrm{IPA}) ; \mathrm{t}_{\mathrm{Rmin}}(\operatorname{anti-3a})=87.4, \mathrm{t}_{\mathrm{Rmaj}}(\operatorname{anti-2a})=111.5 ; \mathrm{t}_{\mathrm{Rmaj}}\left(\right.$ syn-2a)$=149.3, \mathrm{t}_{\mathrm{Rmin}}$ $($ syn-2a) $=193.0$.

1,4-Dihydroxy-4-(4'-nitrophenyl)-2-butanone (iso-3a). ${ }^{60}$ HPLC (Chiralpak AS; $1 \mathrm{~mL} / \mathrm{min}$; $70: 30 \mathrm{Hex} / \mathrm{IPA}) ; \mathrm{t}_{\mathrm{Rmaj}}=12.0, \mathrm{t}_{\mathrm{Rmin}}=16.5$.

4-Hydroxy-3-methoxy-4-(4'-nitrophenyl)-2-butanone (anti-/syn-2b). ${ }^{16 \mathrm{~b}}$ HPLC (Chiracel OD$\mathrm{H} ; 1 \mathrm{~mL} / \mathrm{min} ; 80: 20 \mathrm{Hex} / \mathrm{IPA}) ; \mathrm{t}_{\mathrm{Rmaj}}(\operatorname{anti-2b})=7.1, \mathrm{t}_{\mathrm{Rmin}}(\operatorname{anti-2b})=7.8 ; \mathrm{t}_{\mathrm{Rmin}}($ syn-2b $)=8.1, \mathrm{t}_{\mathrm{Rmaj}}$ $(\operatorname{syn}-\mathbf{2 b})=9.7$. 
4-Hydroxy-1-methoxy-4-(4'-nitrophenyl)-2-butanone (iso-3b). ${ }^{60}$ HPLC (Chiracel OD-H; 1 $\mathrm{mL} / \mathrm{min} ; 80: 20 \mathrm{Hex} / \mathrm{IPA}) ; \mathrm{t}_{\mathrm{Rmin}}=13.4, \mathrm{t}_{\mathrm{Rmaj}}=15.8$.

anti-3-Benzyloxy-4-hydroxy-4-(4'-nitrophenyl)-2-butanone (anti-2c). Yellow oil; $\mathrm{R}_{\mathrm{f}}=0.43$ (Hexane/Ethyl acetate 3:2); IR (neat): $v=3396$ br, 2295, 1730, 1706, 1527, 1347, 1099, 1017 $\mathrm{cm}^{-1} .{ }^{1} \mathrm{H}$ NMR $\left(400 \mathrm{MHz}, \mathrm{CDCl}_{3}\right): \delta=2.17$ (s, 3H, $\mathrm{CH}_{3}$ ), 3.39 (br s, $\left.1 \mathrm{H}, \mathrm{OH}\right), 3.98(\mathrm{~d}, 1 \mathrm{H}, J=5$ $\left.\mathrm{Hz}, \mathrm{HCOCH}_{2} \mathrm{Ph}\right), 4.07\left(\mathrm{~s}, 1 \mathrm{H}, \mathrm{OCH}_{2} \mathrm{Ph}\right), 4.60\left(\mathrm{~s}, 1 \mathrm{H}, \mathrm{OCH}_{2} \mathrm{Ph}\right), 5.28(\mathrm{t}, 1 \mathrm{H}, J=5.2 \mathrm{~Hz}, H \mathrm{COH})$, $7.11(\mathrm{~m}, 1 \mathrm{H}, \mathrm{ArH}), 7.25$ (m, 4H, ArH), 7.55 (d, 2H, $J=8.7 \mathrm{~Hz}, \mathrm{ArH}), 8.18$ (d, 2H, J = 8.7 Hz, $\mathrm{ArH}) .{ }^{13} \mathrm{C} \mathrm{NMR}\left(75 \mathrm{MHz}, \mathrm{CDCl}_{3}\right): \delta=25.3\left(\mathrm{CH}_{3}\right), 29.7\left(\mathrm{CH}_{2}\right), 64.4\left(\mathrm{HCOCH}_{2} \mathrm{Ph}\right), 68.8$ $(\mathrm{CHOH}), 73.6(\mathrm{CHOH}), 123.5,123.8,126.4,127.0,127.9,128.2,128.3,128.55,128.6$ (ArC), $149.9(\mathrm{C}=\mathrm{O})$. HRMS(DIP) $(\mathrm{m} / \mathrm{z})$ : Calcd for $\left(\mathrm{M}^{+}-\mathrm{H}_{2} \mathrm{O}\right): 297.1001$; found: 297.0974; HPLC (Chiracel OD-H; $1 \mathrm{~mL} / \mathrm{min} ; 93: 7 \mathrm{Hex} / \mathrm{IPA}) ; \mathrm{t}_{\mathrm{Rmin}}=27.0, \mathrm{t}_{\mathrm{Rmaj}}=34.2$.

syn-3-Benzyloxy-4-hydroxy-4-(4'-nitrophenyl)-2-butanone (syn-2c). Pale yellow oil; $\mathrm{R}_{\mathrm{f}}=0.55$ (Hexane/Ethyl acetate 3:2); IR (neat): $v=3435 b r, 2924,1715,1605,1522,1347,1216,1110 \mathrm{~cm}^{-}$ 1. ${ }^{1} \mathrm{H}$ NMR (400 MHz, $\left.\mathrm{CDCl}_{3}\right): \delta=2.16\left(\mathrm{~s}, 3 \mathrm{H}, \mathrm{CH}_{3}\right), 3.13($ br s, $1 \mathrm{H}, \mathrm{OH}), 3.90(\mathrm{~d}, 1 \mathrm{H}, J=6.4$ $\left.\mathrm{Hz}, \mathrm{HCOCH}_{2} \mathrm{Ph}\right), 4.30\left(\mathrm{~d}, 1 \mathrm{H}, J=11.5 \mathrm{~Hz}, \mathrm{OCH}_{2} \mathrm{Ph}\right), 4.51\left(\mathrm{~d}, 1 \mathrm{H}, J=11.5 \mathrm{~Hz}, \mathrm{OCH}_{2} \mathrm{Ph}\right), 5.03$ (d, 1H, $J=8 \mathrm{~Hz}, H \mathrm{COH}), 7.15(\mathrm{~m}, 1 \mathrm{H}, \mathrm{ArH}), 7.31$ (m, 4H, ArH), 7.54 (d, 2H, J = 8.7 Hz, ArH), 8.19 (d, 2H, $J=8.7 \mathrm{~Hz}, \mathrm{ArH}) .{ }^{13} \mathrm{C} \mathrm{NMR}\left(75 \mathrm{MHz}, \mathrm{CDCl}_{3}\right): \delta=27.6\left(\mathrm{CH}_{3}\right), 29.7\left(\mathrm{CH}_{2}\right), 73.5$ $\left(\mathrm{HCOCH}_{2} \mathrm{Ph}\right), 83.9(\mathrm{CHOH}), 123.4,123.8,126.4,127.0,127.7,128.1,128.4,128.6,136.2$ $(\mathrm{ArC}), 146.8(\mathrm{C}=\mathrm{O})$. HRMS(DIP) $(\mathrm{m} / \mathrm{z})$ : Calcd for $\left(\mathrm{M}-\mathrm{H}_{2} \mathrm{O}\right)$ : 297.1001; found: 297.1022; HPLC (Chiracel OD-H; $1 \mathrm{~mL} / \mathrm{min} ; 93: 7 \mathrm{Hex} . / \mathrm{IPA}) ; \mathrm{t}_{\mathrm{Rmaj}}=29.9, \mathrm{t}_{\mathrm{Rmin}}=31.9$.

iso-1-Benzyloxy-4-hydroxy-4-(4'-nitrophenyl)-2-butanone (3c). Pale yellow oil; $\mathrm{R}_{\mathrm{f}}=0.52$ (hexane/ethyl acetate 3:2); IR (neat): $v=3418 \mathrm{br}, 2918,1727,1596,1516,1361,1250,1105 \mathrm{~cm}^{-}$ 1. ${ }^{1} \mathrm{H}$ NMR $\left(400 \mathrm{MHz}, \mathrm{CDCl}_{3}\right): \delta=2.92\left(\mathrm{t}, 2 \mathrm{H}, J=2.7 \mathrm{~Hz}, \mathrm{CH}_{2} \mathrm{CHOH}\right), 4.07(\mathrm{~s}, 2 \mathrm{H}$, $\left.\mathrm{CH}_{2} \mathrm{OCH}_{2} \mathrm{Ph}\right), 4.53\left(\mathrm{~s}, 2 \mathrm{H}, \mathrm{OCH}_{2} \mathrm{Ph}\right), 4.61(\mathrm{~d}, 1 \mathrm{H}, J=11.5 \mathrm{~Hz}, H \mathrm{COH})$, ), $5.08(\mathrm{~m}, 1 \mathrm{H}, H \mathrm{COH})$, $7.13(\mathrm{~m}, 1 \mathrm{H}, \mathrm{ArH}), 7.27$ (m, 4H, ArH), 7.54 (d, 2H, $J=8.7 \mathrm{~Hz}, \mathrm{ArH}), 8.17$ (d, 2H, $J=8.7 \mathrm{~Hz}$, $\mathrm{ArH}) .{ }^{13} \mathrm{C}$ NMR $\left(75 \mathrm{MHz}, \mathrm{CDCl}_{3}\right): \delta=47.5\left(\mathrm{CH}_{2} \mathrm{CHOH}\right), 68.8(\mathrm{CHOH}), 73.6\left(\mathrm{CH}_{2} \mathrm{Ph}\right), 75.2$ $\left(\mathrm{OCH}_{2} \mathrm{C}=\mathrm{O}\right), 123.8,126.4,128.0,128.3,128.4,128.6,135.9$ (ArC), $150.0(\mathrm{C}=\mathrm{O})$. HRMS(DIP) $(\mathrm{m} / \mathrm{z})$ : Calcd for $\left(\mathrm{M}-\mathrm{C}_{7} \mathrm{H}_{7}\right)$ : 225.0637; found: 225.0663; HPLC (Chiralpak AD; $1.2 \mathrm{~mL} / \mathrm{min}$; 97:3 Hex/IPA); $\mathrm{t}_{\mathrm{Rmaj}}=190.9, \mathrm{t}_{\mathrm{Rmin}}=205.1$.

4-Hydroxy-3-(methylsulphanyl)-4-(4'-nitrophenyl)-2-butanone $\quad\left(\right.$ anti-/syn-2d) ${ }^{16 \mathrm{~b}} \quad$ HPLC $($ Chiracel OD-H; $1 \mathrm{~mL} / \mathrm{min} ; 88: 12 \mathrm{Hex} / \mathrm{IPA}) ; \mathrm{t}_{\mathrm{Rmaj}}=11.6, \mathrm{t}_{\mathrm{Rmin}}(\operatorname{anti-2d})=14.1 ; \mathrm{t}_{\mathrm{Rmin}}(\operatorname{syn}-\mathbf{2 d})=$ $13.4, \mathrm{t}_{\mathrm{Rmaj}}(\operatorname{syn}-\mathbf{2 d})=14.9$.

4-Hydroxy-1-(methylsulphanyl)-4-(4'-nitrophenyl)-2-butanone (iso-3d) ${ }^{16}$ HPLC (Chiracel OD-H; $1 \mathrm{~mL} / \mathrm{min} ; 88: 12 \mathrm{Hex} / \mathrm{IPA}) ; \mathrm{t}_{\mathrm{Rmin}}=16.6, \mathrm{t}_{\mathrm{Rmaj}}=17.9$. 


\section{Acknowledgements}

This work was financially supported by the Dirección General de Investigación of the Ministerio de Educación y Ciencia of Spain (Grant CTQ2004-00808/BQU), the Generalitat Valenciana (Grant CTIOIB/2002/320, GRUPOS03/134 and GV05/157) and the University of Alicante.

\section{References}

1. (a) Dalko, P. I.; Moisan, L. Angew. Chem. Int. Ed. 2001, 40, 3726. (b) Benaglia, M.; Puglivi, A.; Cozzi, F. Chem. Rev. 2003, 103, 3401. (c) List, B.; Bolm, C.; Issue Eds. Ad. Synth. Catal. 2004, 346, 1021 (thematic issue on Organic Catalysis, no. 9+10). (d) Houk, K. N.; List, B. Guest Eds. Acc. Chem. Res. 2004, 37, 487 (thematic issue on Asymmetric Organocatalysis, no. 8). (e) Dalko, P. I.; Moisan, L. Angew. Chem. Int. Ed. 2004, 43, 5138. (f) Berkessel, A.; Gröger, H. Asymmetric Organocatalysis: From Biomimetic Concepts to Applications in Asymmetric Synthesis; Wiley-VCH: Weinheim, 2005. (g) Seayad, J.; List, B. Org. Biomol. Chem. 2005, 3, 719. (h) Kočovský, P.; Malkov, A. V. Issue Eds. Tetrahedron 2006, 62, 243 (thematic issue on Organocatalysis in Organic Synthesis, no. 2-3).

2. (a) Marigo, M.; Jørgensen, K. A. Chem. Commun. 2006, 2001. (b) Guillena, G.; Ramón, D. J. Tetrahedron: Asymmetry 2006, 17, 1465.

3. Mogassaghi, M.; Jacobsen, E. N. Science 2002, 1904.

4. (a) For general reviews on the catalytic enantioselective aldol reaction, see: (a) Gröger, H.; Vogl, E. M.; Shibasaki, M. Chem. Eur. J. 1998, 4, 1137. (b) Nelson, S. G. Tetrahedron: Asymmetry 1998, 9, 357. (c) Carreira E. M. In Comprehensive Asymmetric Catalysis, Jacobsen, E. N.; Platz, A.; Yamamoto, H. Eds.; Springer: Heidelberg, 1999, Vol. 3, Chap 29, pp 997. (d) Mahrwald, R. Chem. Rev. 1999, 96, 1095. (e) Machajewski, T. D.; Wong, C.-H. Angew. Chem. Int. Ed. 2000, 39, 1352. (f) Alcaide, B.; Almendros, P. Eur. J. Org. Chem. 2002, 1595. (g) Modern Aldol Reactions, Vols 1-2, Marhrwald, R. Ed.; Wiley-VCH: Weinheim, 2004. (h) Palomo, C.; Oiarbide, M.; García, J. M. Chem. Soc. Rev. 2004, 33, 65. (i) Mestres, R. Green Chem. 2004, 6, 583.

5. For the first proline catalyzed intramolecular reaction see: (a) List, B.; Lerner, R. A.; Barbas III, C. F. J. Am. Chem. Soc. 2000, 122, 2395. For recent uses of proline in the direct aldol reaction, see: (b) Córdova, A.; Ibrahem, I.; Casas, J.; Sundén, H.; Engqvist, M.; Reyes, E. Chem. Eur. J. 2005, 11, 4772. (c) Reyes, E.; Córdova, A. Tetrahedron Lett. 2005, 60, 6605. (d) Liao, W.-W.; Ibrahem, I.; Córdova, A. Chem. Commun. 2006, 674. (e) Ibrahem, I.; Zou, W.; Xu, Y.; Córdova, A. Adv. Synth. Catal. 2006, 348, 211. (f) Kuchurenko, A. S.; Struchkova, M. I.; Zlotin, S. G. Eur. J. Org. Chem. 2006, 2000. (g) Suri, J. T.; Mitsumori, S.; Albertshofer, K.; Tanaka, F.; Barbas III, C. F. J. Org. Chem. 2006, 71, 3822. (h) Kumar, I.; Rode, C. V. Tetrahedron: Asymmetry 2006, 17, 763. (i) Samanta, S.; Zhao, C. G. Tetrahedron Lett. 2006, 47, 3383. 
6. (a) Saito, S.; Nakadai, M.; Yamamoto, H. Synlett 2001, 1245. (b) Nakadai, M.; Saito, S.; Yamamoto, H. Tetrahedron 2002, 58, 1245. (c) Tang, Z.; Jiang, F.; Yu, L.-T.; Mi, A.-Q.; Jiang, Y.-Z.; Wu, Y.-D. J. Am. Chem. Soc. 2003, 125, 5262. (d) Kofoed, J.; Nielsen, J.; Reymond, J. L. Bioorg. Med. Chem. Lett. 2003, 13, 2445. (e) Martin, H. J.; List, B. Synlett 2003, 1901. (f) Tanimori, S.; Naka, T.; Kirihata, M.; Synth. Commun. 2004, 34, 4043. (g) Tang, Z.; Jiang, F.; Cui, X.; Gong, L.-Z.; Mi, A.-Q.; Jiang, Y.-Z.; Wu, Y.-D. Proc. Natl. Acad. Sci. U. S. A. 2004, 101, 5755. (h) Torii, H.; Nakadai, M.; Ishihara, K.; Saito, S.; Yamamoto, H. Angew. Chem. Int. Ed. 2004, 43, 1983. (i) Lacoste, E.; Landais, Y.; Schenk, K.; Verlhac, J.-B.; Vincent, J.-M. Tetrahedron Lett. 2004, 45, 8035. (j) Berkessel, A.; Koch, B.; Lex, J. Adv. Synth. Catal. 2004, 346, 1141. (k) Shi, L.-X.; Sun, Q.; Ge, Z.-M.; Zhu, Y.Q.; Cheng, T.-M.; Li, R.-T. Synlett 2004, 2215. (1) Guo, H.-M.; Cun, L-F.; Gong, L.-Z.; Mi, Y. A. Q. Chem. Commun. 2005, 1450. (m) Tang, Z.; Yang, Z.-H.; Chen, L-F.; Cun, X.-H.; Mi, A.-Q.; Jiang, Y.-Z.; Gong, L.-Z. J. Am. Chem. Soc. 2005, 127, 9285. (n) Cobb, J. A.; Shaw, M.; Longbottom, A.; Gold, J. B.; Ley, S. V. Org. Biomol. Chem. 2005, 3, 84. (o) Tang, Z.; Yang, Z.-H.; Cun, L.-F.; Gong, L.-Z.; Mi, A.-Q.; Jiang, Y.-Z. Org. Lett. 2004, 6, 2285. (p) Krattiger, P.; Kovasy, R.; Revell, J. D.; Ivan, S.; Wennemers H. Org. Lett. 2005, 7, 1101. (q) Zhang, F., Su, W.; Gong, Y. Synlett 2006, 1703. (r) Dinér, P.; Amedjkouh, M. Org. Biomol. Chem. 2006, 4, 2091. (s) Gu, Q.; Wang, X.-F.; Wang, L.; Wu, X.-Y.; Zhou, Q.-L. Tetrahedron: Asymmetry 2006, 17, 1537. (t) Raj, M.; Vishnumaya; Ginotra, S. K.; Singh, V. K. Org. Lett. 2006, 8, 4097.

7. (a) Hayashi, Y.; Sumiya, T.; Takahashi, J.; Gotoh, H.; Urushima, T.; Shoji, M. Angew. Chem. Int. Ed. 2006, 45, 958. (b) Mase, N.; Nakai, Y.; Ohara, N.; Yoda, H.; Takabe, K.; Tanaka, F.; Barbas II, C. F. J. Am. Chem. Soc. 2006, 128, 734. (c) Dziedzic, P.; Zou, W.; Háfren, J.; Córdova, A. Org. Biomol. Chem. 2006, 4, 38. (d) Hayashi, Y.; Aratake, S.; Okano, T.; Takahashi, J.; Sumiya, T.; Shoji, M. Angew. Chem. Int. Ed. 2006, 45, 5527.

8. (a) Notz, W.; List, B. J. Am. Chem. Soc. 2000, 122, 7386. (b) Pan, Q.; Zou, B.; Wang, Y.; Ma, D. Org. Lett. 2004, 6, 1009. (c) Calderón, F.; Fernández, R.; Sánchez, F.; FernándezMayorales, A. Adv. Synth. Catal. 2005, 347, 1389.

9. (a) Liu, H.; Peng, L.; Li, Y. New. J. Chem. 2003, 27, 1159. (b) Kitazume, T.; Jiang, Z.; Kasai, K.; Mihara, Y.; Suzuki, M. J. Fluor. Chem. 2003, 121, 205. (c) Peng, L.; Liu, H.; Zhang, T.; Zhang, F.; Mei, T.; Li, Y.; Li, Y. Tetrahedron Lett. 2003, 44, 5107. (d) Zhong, G.; Fan, J.; Barbas III, C. F. Tetrahedron Lett. 2004, 45, 5681. (e) He, L.; Tang, Z.; Cun, L.F.; Mi, A.-Q.; Jiang, Y.-Z.; Gong, L.-Z. Tetrahedron 2006, 62, 346.

10. Guillena, G.; Hita, M. C.; Nájera, C. Tetrahedron: Asymmetry 2006, 17, 729.

11. Gryko, D.; Kowalczyk, B.; Zawadzki, L. Synlett 2006, 1059.

12. Guillena, G.; Hita, M. C.; Nájera, C. Tetrahedron: Asymmetry 2006, 17, 1027.

13. Guillena, G.; Hita, M. C.; Nájera, C. Tetrahedron: Asymmetry 2006, 17, 1493.

14. (a) K. Mislow, P. A. Graseman, J. Org. Chem. 1958, 23, 2027. (b) For the synthesis and structural properties of several BINAM prolinamides derived from acyclic amino acids see: Kowalczyk, B.; Tarnowska, A.; Weseliński, L; Jurczak, J. Synlett 2005, 2372. (c) For the 
use of BINAM-prolinamides in other reactions, see: Xiong, Y.; Huang, X.; Gou, S.; Huang, J.; Wen, Y.; Feng, X. Adv. Synth. Catal. 2006, 348, 538.

15. For review about the use of $\alpha$-hydroxyacetone derivatives in organic synthesis, see: Enders, D.; Voith, M.; Lenzen, A. Angew. Chem. Int. Ed. 2005, 44, 1304

16. (a) Maggiotti, V.; Resmini, M.; Gouverneur, V. Angew. Chem. Int. Ed. 2002, 41, 1012. (b) Baker-Glenn, C.; Ancliff, R.; Gouverneur, V. Tetrahedron 2004, 60, 7607. 